\title{
Effects of electron-hole separation on the photoconductivity of individual metal oxide nanowires
}

\author{
J D Prades ${ }^{*, 1}$, F Hernandez-Ramirez ${ }^{* 1,2}$, R Jimenez-Diaz ${ }^{1}$, M Manzanares ${ }^{1}$, \\ T Andreu ${ }^{1}$, A Cirera ${ }^{1}$, A Romano-Rodriguez ${ }^{1}$ and $\mathbf{J}$ R Morante ${ }^{1}$ \\ ${ }^{1} \mathrm{EME} / \mathrm{XaRMAE} / \mathrm{IN}^{2} \mathrm{UB}$, Departament d'Electrònica, Universitat de Barcelona, \\ C/ Martí i Franquès 1, E-08028 Barcelona, Spain \\ ${ }^{2}$ Electronic Nanosystems S. L., Barcelona, Spain \\ E-mail: dprades@el.ub.es, fhernandez@gmail.com
}

\begin{abstract}
Responses of individual $\mathrm{ZnO}$ nanowires to UV light demonstrate that the Persistent PhotoConductivity (PPC) state is directly related to the electron-hole separation near the surface. Our results demonstrate that the electrical transport in these nanomaterials is influenced by the surface in two different ways. On the one hand, the effective mobility and the density of free carriers are determined by recombination mechanisms assisted by the oxidizing molecules in air. This phenomenon can also be blocked by surface passivation. On the other hand, the surface built-in potential separates the photogenerated electron-hole pairs and accumulates holes at the surface. After illumination, the charge separation makes difficult the electron-hole recombination and originates PPC. This effect is quickly reverted after increasing either the probing current (self-heating by Joule dissipation) or the oxygen content in air (favouring the surface recombination mechanisms). The here-presented model for PPC in individual nanowires illustrates the intrinsic potential of metal oxide nanowires to develop optoelectronic devices or optochemical sensors with better and new performances.
\end{abstract}

\section{Introduction}

Methods for synthesis of nanomaterials have undergone a huge improvement in the last years $[1,2]$, providing researchers with nanowires with high surface-to-volume ratio and excellent crystalline properties $[3,4]$. Their electrical characterization is a direct way to gain a deep comprehension of some of the phenomena typical of the nanoscale, which are originated due to the overexposure of the bulk of nanomaterials to surface effects [5,6,7]. To date, the large majority of works have focused on the study of nanowire bundles [8], but uncontrolled parasitic effects between nanowires has made difficult obtaining any conclusive result [9]. The constant improvement of nanofabrication techniques is slowly changing this situation, enabling the appearance of many works dealing with individual nanowires, and whose electrical characterization is extremely helpful to evaluate transport phenomena in single-crystal structures [10].

Among nanowires, metal-oxide ones are excellent candidates to evaluate the influence of surface effects on the bulk properties of nanomaterials, since they are highly reactive to their surrounding atmosphere [11]. This property has attracted an increasing interest to integrate them as building blocks of new nanodevices, like gas sensors or optoelectronic devices [6,7]. Zinc oxide $(\mathrm{ZnO})$ nanowires are versatile metal-oxide nanomaterials which have been successfully tested in different fields [12], like chemical [3] and biological sensing [13], optoelectronics [6,7], energy harvesting [14], and ultraviolet (UV) photodetection [15-19]. In this last case, they even exhibit higher photoconductive gains $[18,19]$ than equivalent devices based on thin film technologies [20].

\footnotetext{
* Authors to whom any correspondence should be addressed.
} 
The effects of UV light on $\mathrm{ZnO}$ are more complex than a simple band-to-band photoresponse [15-19], since it often induces a persistent increase of the electrical conductivity which lasts for a long time after the exposure to UV [21-30] and requires a post-treatment of the sample to recover the initial value. The origin of this Persistent PhotoConductivity (PPC) phenomenon is still controversial. On the one hand, some authors relate PPC to either intrinsic or extrinsic point defects which exhibit metastable charge states [21-23]. On the other hand, others claim that the origin is the electron-hole separation related to the surface properties of metal oxides [24-27,29,30]. Thus, a widely accepted description has not been presented yet [23].

In this paper, the strong influence of surface effects on the transport phenomena of nanomaterials has been used to evaluate the PPC effect in individual $\mathrm{ZnO}$ nanowires and demonstrate its surface origin. Unlike previous works, here, the use of a single nanowire has allowed to exclude other influences and experimentally simplify the analysis. We have studied the influence of the surface recombination mechanisms on PPC via the effective mobility and the density of free carriers. Moreover, the PPC recovery at different working conditions (probing currents and atmospheres) has also been studied in detail. Based on these results, a model for PPC in a single nanowire is presented, and different strategies to minimize or even block the PPC in $\mathrm{ZnO}$ are outlined, enabling the design of nanowire-based photodetectors free of this undesirable effect.

\section{Experimental details}

$\mathrm{ZnO}$ nanowires [3,31] (see supporting information) were dispersed in propylenglycol and deposited onto an insulating $\mathrm{SiO}_{2}$ chip with pre-patterned $\mathrm{Au} / \mathrm{Ti} / \mathrm{Ni}$ microelectrodes. Then, some of them were electrically contacted to the microelectrodes in 4-probe configuration with a FEI Dual-Beam Strata 235 Focused Ion Beam (FIB) equipped with a metalorganic precursor to deposit Pt strips (figure 1) [32], following a methodology designed to minimize structural modification during the fabrication process [9]. The electrical response of the final devices was evaluated inside a home-made chamber specially designed to illuminate the sample at the same time than different atmospheres were applied with the help of four gas mass-flow systems. The light power impinging on this sample $(\lambda=340 \pm 10 \mathrm{~nm})$ was estimated with a photodiode located beside it [33]. A home-made electronic circuit designed to guarantee that low current levels $I_{m}$ (from $0.1 \mathrm{nA}$ to $250 \mathrm{nA}$ ) were applied during the experiments was used [34], and parasitic effects of the metal-nanowire contacts were avoided performing 4-probing DC measurements [10] (see supporting information). Fifteen of these devices exhibited stable characteristics without any noticeable degradation after three weeks of continuous operation. All the experiments were performed in either synthetic air (SA) or nitrogen $\left(\mathrm{N}_{2}\right)$. Finally, some of them were passivated with a $470 \mathrm{~nm}$ thick PMMA layer deposited by spin coating. This layer was UV transparent and electrically insulating (see supporting information).

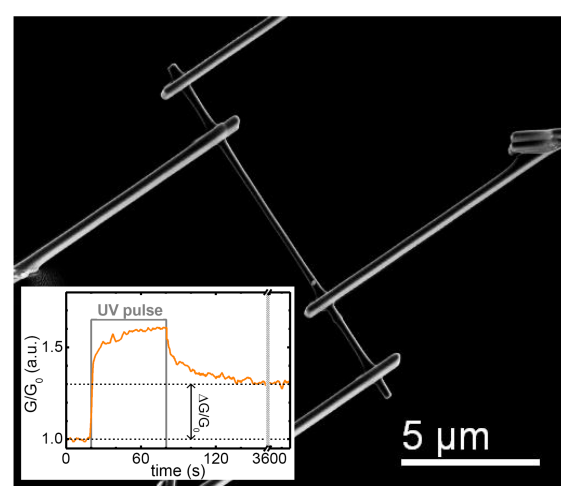

Figure 1. $\mathrm{ZnO}$ nanowire of length $\mathrm{L}_{\mathrm{NW}}=13 \mu \mathrm{m}$ and radius $\mathrm{r}_{\mathrm{NW}} \sim 95 \mathrm{~nm}$ electrically contacted with FIB nanolithography techniques. (Inset) Evidence of PPC in these nanowires: the conductance baseline was not fully recovered after switching off the light. 


\section{Experimental Results and Discussion}

\subsection{Evidence of PPC in individual $\mathrm{ZnO}$ nanowires}

Non illuminated $\mathrm{ZnO}$ nanowires (length $\mathrm{L}_{\mathrm{NW}}=13 \mu \mathrm{m}$ and radius $\mathrm{r}_{\mathrm{NW}} \sim 95 \mathrm{~nm}$ ) in oxygen rich atmospheres exhibited conductance values of $\mathrm{G}_{0(\mathrm{SA})}=59 \pm 6 \mathrm{nS}$, which are in agreement with reported data [15-19] (see supporting information). No significant photoresponse was observed with impinging photons with energies hv below the bandgap of $\mathrm{ZnO}\left(\mathrm{E}_{\text {gap }}=3.37 \mathrm{eV}\right.$ [35]) (see supporting information). On the contrary, photons with higher energies lead to an important increase of the electrical conductance of nanowires. This result confirms that photoconduction in $\mathrm{ZnO}$ nanowires is mainly originated by band-to-band electron-hole pair generation in their bulk. Dynamic measurements showed that the conductance baseline was not fully recovered after switching off the light, demonstrating the existence of PPC in these nanomaterials (inset in figure 1).
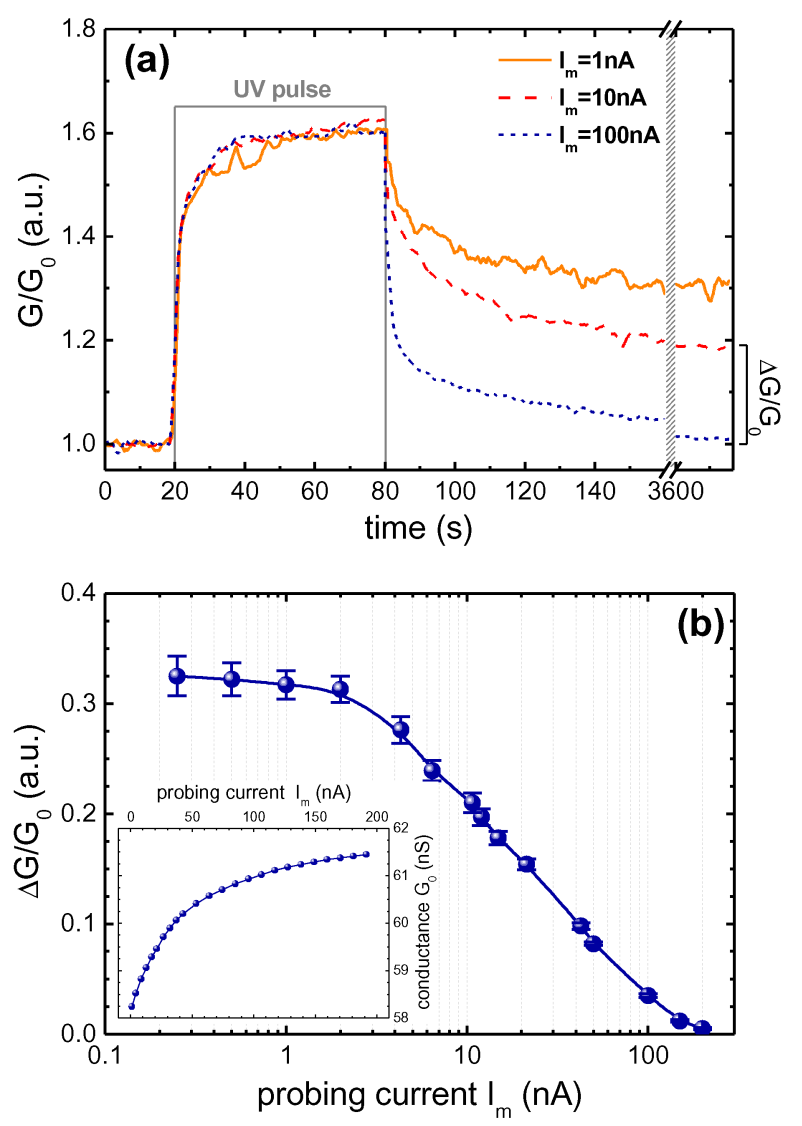

Figure 2. (a) Relative conductance change $\left(\mathrm{G} / \mathrm{G}_{0}\right)$ of a $\mathrm{ZnO}$ nanowire under illumination $(60 \mathrm{~s}$ UV pulse, photon flux $\Phi_{\mathrm{ph}}=3.3 \cdot 10^{18} \mathrm{~m}^{-2} \mathrm{~s}^{-1}$, wavelength $\lambda=340 \pm 10 \mathrm{~nm}$ ) acquired at three different current levels $\left(\mathrm{I}_{\mathrm{m}}\right)$ in synthetic air. Axis break shows the conductance one hour later. (b) Dependence of the PPC with the probing current $\mathrm{I}_{\mathrm{m}}$ in synthetic air. The magnitude of the PPC $\left(\Delta \mathrm{G} / \mathrm{G}_{0}\right)$ was determined as described in the text. PPC values and error bars shown in the figure are the mean and the standard deviation of the results obtained with ten different samples. The dependence of the conductance in dark $\left(\mathrm{G}_{0}\right)$ with the probing current is shown in the inset. Conductance rise demonstrates the self-heating of the nanowires during the measurement, in accordance with the semiconductor character of $\mathrm{ZnO}$. 

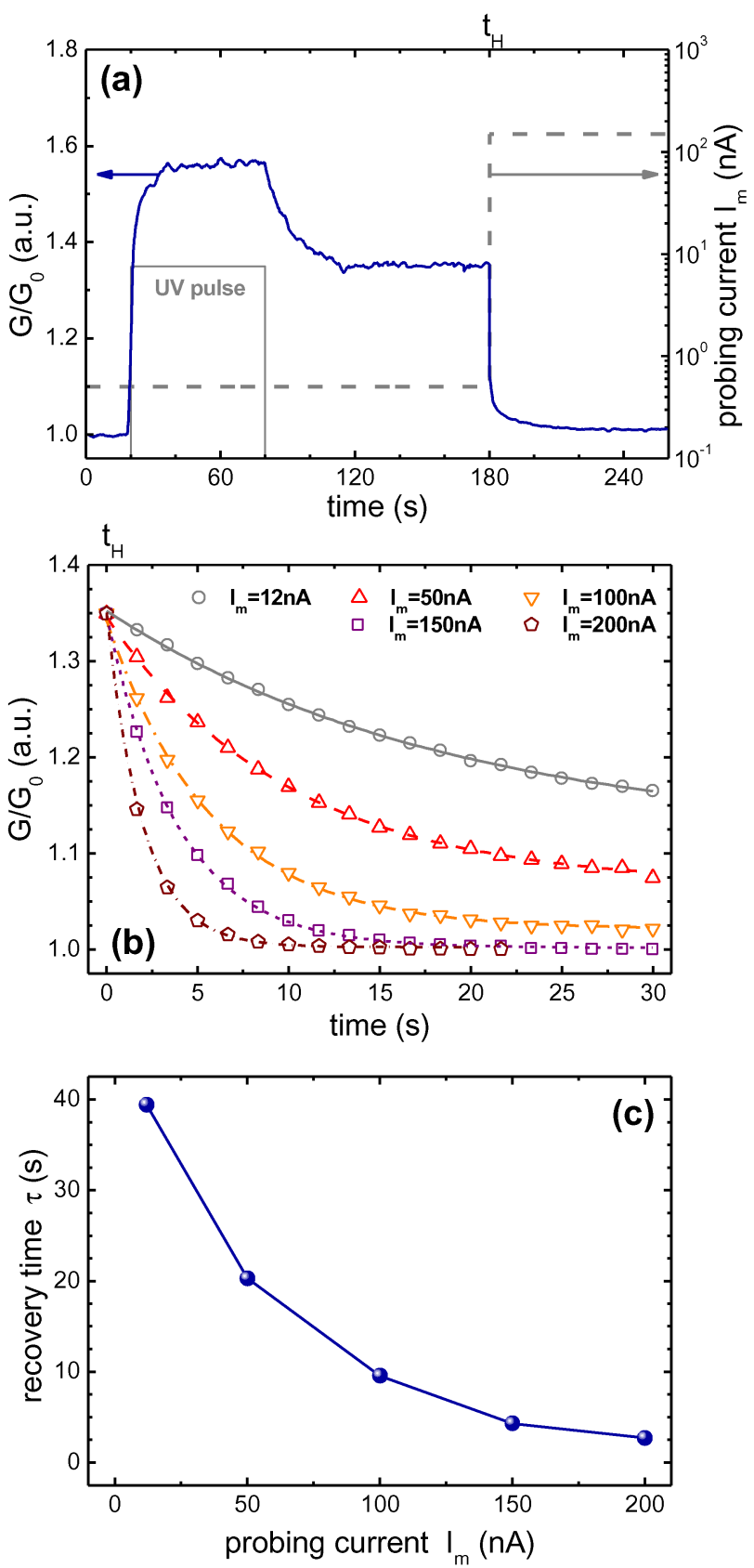

Figure 3. Recovery of the PPC stimulated by high probing current values. (a) $G / G_{0}$ record. A $60 \mathrm{~s}$ UV pulse was applied to a $\mathrm{ZnO}$ nanowire in low current conditions $\left(\mathrm{I}_{\mathrm{m}}=0.5 \mathrm{nA}\right)$. After the stabilization of the PPC $\left(\right.$ at $\left.t_{H}\right)$, the conductance recovery was stimulated by increasing the probing current $\left(\mathrm{I}_{\mathrm{m}}=150 \mathrm{nA}\right)$. (b) Analysis of the recovery transients for different $\mathrm{I}_{\mathrm{m}}$. Experimental data points fit to exponential decay laws (lines) to estimate the recovery time constants $\tau$. (c) PPC recovery time constant $\tau$ as function of the probing current $\mathrm{I}_{\mathrm{m}}$.

\subsection{Influence of the probing current on PPC}

It was experimentally found that the intensity of PPC is directly related to the probing current $\left(\mathrm{I}_{\mathrm{m}}\right)$ applied to the nanowires (figure 2). The recovery of the conductance $\mathrm{G}$ of a $\mathrm{ZnO}$ nanowire after a 60 second UV pulse at different $\mathrm{I}_{\mathrm{m}}$ is shown in figure $2 \mathrm{a}$. Here, the relative persistence of the conductance was defined as, 


$$
\frac{\Delta \mathrm{G}}{\mathrm{G}_{0}}=\frac{\mathrm{G}_{1 \mathrm{~h}}-\mathrm{G}_{0}}{\mathrm{G}_{0}}
$$

where $\mathrm{G}_{0}$ was the initial value in darkness and $\mathrm{G}_{\mathrm{lh}}$ one hour after the pulse.

The PPC effect fully disappeared one hour after illuminating the sample with $\mathrm{I}_{\mathrm{m}}>100 \mathrm{nA}$. On the contrary, PPC did not vanish with lower $\mathrm{I}_{\mathrm{m}}$, reaching a maximum with $\mathrm{I}_{\mathrm{m}}<1 \mathrm{nA}$ (figure $2 b$ ). These two different behaviours are an indirect proof of the well-known dependence on PPC with temperature $[21,25,26,36]$, since nanodevices reach high temperatures due to the dissipated electrical power when they come into operation. To validate this assumption, the recovery of $\mathrm{G}$ in a $\mathrm{ZnO}$ nanowire showing PPC was stimulated with a sudden increase of $\mathrm{I}_{\mathrm{m}}$ from $0.5 \mathrm{nA}$ to $150 \mathrm{nA}$ (figure 3a). Transient responses in good correspondence with exponential decays were found in all the experiments (figure $3 b$ ). The higher the probing current $I_{m}$ was applied to the nanodevices, the faster the recovery time was observed (figure 3c). Equivalent responses were monitored in SA and $\mathrm{N}_{2}$. Thus, the modulation of $\mathrm{I}_{\mathrm{m}}$ is proposed as a simple and fast methodology to control PPC, despite it does not shed light on the origin of PPC in $\mathrm{ZnO}$. It goes without saying that an accurate estimation of the temperature reached by the nanowire due to Joule self-heating would provide valuable information about the activation energies of the states related to PPC, but this point remains as a complex experimental issue [37].

\subsection{On the origin of PPC in $\mathrm{ZnO}$ nanowires}

There are two opposite models which describe PPC in $\mathrm{ZnO}$. The first one claims that this phenomenon is related to metastable bulk defects located between their shallow and deep energy levels [21-23]. According to this assumption, oxygen vacancies can be excited to a metastable charged state after a structural relaxation. In these states, the recapture of electrons is prevented by a thermally activated barrier [21]. The second one upholds that PPC is a pure surface effect related to the capture of electrons by surface states [26,27,29], which arise with the generation of oxygen vacancies by UV light [24,26,30]. This process is also reverted by temperature-assisted adsorption of oxygen species [26-28]. In these two theoretical approaches, if electron-hole pairs are spatially separated, their recombination rate is significantly reduced, giving rise to PPC.

\section{3. a Evaluation of surface effects in PPC}

To clarify the origin of PPC, individual $\mathrm{ZnO}$ nanowires were used to take advantage of the overexposure of their bulk to surface effects. $\mathrm{I}_{\mathrm{m}}=1 \mathrm{nA}$ was always applied, and the experiments were systematically repeated in both SA and $\mathrm{N}_{2}$ (with $50 \mathrm{ppm}$ of residual $\mathrm{O}_{2}$ ). Conductance modulation curves were represented in terms of $\left\{\left(\mathrm{G} / \mathrm{G}_{0}\right)-1\right\}$ to facilitate the comparison of the different recovery transients.

In figure $4 \mathrm{a}, \mathrm{G}_{0}$ of a $\mathrm{ZnO}$ nanowire was respectively $\mathrm{G}_{0(\mathrm{SA})}=59 \pm 6 \mathrm{nS}$ and $\mathrm{G}_{0(\mathrm{~N} 2)}=110 \pm 10 \mathrm{nS}$ in $\mathrm{SA}$ and $\mathrm{N}_{2}$. UV illumination gave rise to photoresponses 4.2 times higher in $\mathrm{N}_{2}$ than in SA. Equivalent responses were monitored with the rest of nanowires, confirming that surface interaction mechanisms dominate UV photoresponse and PPC. To decouple bulk and surface phenomena, some of them were passivated with PMMA. Previous works proposed that coating $\mathrm{ZnO}$ with polymers not only prevents the interaction of its surface with gases, but it also passivates the electron states of metal oxides associated to dangling bonds located at the surface $[19,38-40]$. The conductance in darkness of the coated sample $\left(\mathrm{G}_{0(\mathrm{PMMA})}=1400 \pm 120 \mathrm{nS}\right)$ was 24 times higher than in $\mathrm{SA}\left(\mathrm{G}_{0(\mathrm{SA})}=59 \pm 6 \mathrm{nS}\right)$, whereas photoresponse was found to increase 12 times in comparison to the response in SA. 

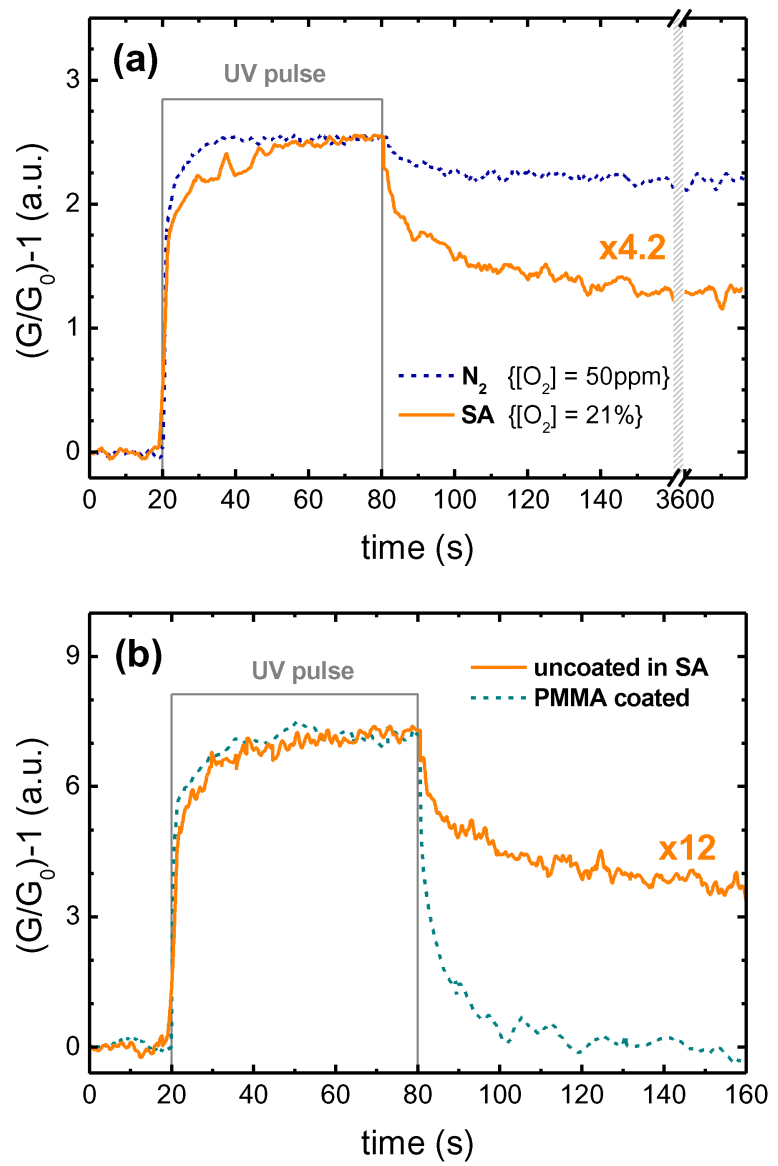

Figure 4. Relative conductance $\left\{\left(\mathrm{G} / \mathrm{G}_{0}\right)-1\right\}$ of a $\mathrm{ZnO}$ nanowire with a $60 \mathrm{~s}$ UV pulse (photon flux $\Phi_{\mathrm{ph}}=3.3 \cdot 10^{18} \mathrm{~m}^{-2} \mathrm{~s}^{-1}$, wavelength $\lambda=340 \pm 10 \mathrm{~nm}$ ) acquired at low-probing current conditions $\left(\mathrm{I}_{\mathrm{m}}=1 \mathrm{nA}\right)$. Solid-orange plots were rescaled (multiplying the data by $\times 4.2$ and $\times 12$, respectively) to ease the comparison of the recovery processes. (a) Two different atmospheres were used: synthetic air (SA) and nitrogen $\left(\mathrm{N}_{2}\right)$. The oxygen concentration was $\left[\mathrm{O}_{2}\right]_{\mathrm{SA}}=210000$ ppm and $\left[\mathrm{O}_{2}\right]_{\mathrm{N} 2}=50 \mathrm{ppm}$, respectively. Response to the UV pulse in $\mathrm{N}_{2}$ was 4.2 times larger than in SA. (b) Relative conductance $\left\{\left(\mathrm{G} / \mathrm{G}_{0}\right)-1\right\}$ of a $\mathrm{ZnO}$ nanowire with the same UV pulse before and after coating the nanostructure with PMMA. Response to the UV pulse after coating was 12 times larger than before.

\section{3.b Modelling PPC in $\mathrm{ZnO}$ nanowires}

The electrical properties of metal oxide nanowires are strongly influenced by the environment. In general, oxygen molecules in air absorb onto their surface trapping conduction electrons from the bulk [41]. This process leads to the appearance of a negative charge distribution fixed at the surface, which is generally described with an upward band bending [41]. In the steady-state, this phenomenon reduces the effective conduction channel along the nanowires and modulates $\mathrm{G}$ as $[42,43]$

$$
G=|e| n_{0} \mu \frac{\pi\left(r_{N W}-\lambda\right)^{2}}{L_{N W}}
$$

where $G$ is the conductance of a nanowire of radius $r_{N w}$ and length $L_{N W}$, e the fundamental charge, $\mathrm{n}_{0}$ the concentration of free carriers, $\mu$ their electrical mobility and $\lambda$ the width of the depletion layer, which depends on the chemisorbed species.

In oxygen-rich atmospheres, $\lambda$ grows and thus, lower electrical conductance is observed. On the contrary, thinner $\lambda$ arises with low pressure oxygen atmospheres and higher conductance values are monitored [42]. This prediction is in agreement with our experimental data: 


$$
\mathrm{G}_{0 \text { (PMMA) }}=1400 \mathrm{nS} \gg \mathrm{G}_{0\left(\mathrm{~N}_{2}\right)}=110 \mathrm{nS}>\mathrm{G}_{0(\mathrm{SA})}=59 \mathrm{nS} .
$$

The $\lambda$ size in $\mathrm{SA}$ for $\mathrm{ZnO}$ nanowires can be estimated by solving the Poisson equation [43],

$$
\mathrm{V}_{\mathrm{b}}=\frac{|\mathrm{e}| \mathrm{n}_{0}}{4 \varepsilon_{0} \varepsilon_{\mathrm{r}}}\left[\left(2 \mathrm{r}_{\mathrm{NW}}^{2}-\lambda^{2}\right)-2\left(\mathrm{r}_{\mathrm{NW}}-\lambda\right)^{2} \log \left(\mathrm{r}_{\mathrm{NW}} /\left(\mathrm{r}_{\mathrm{NW}}-\lambda\right)\right)\right] \text {. }
$$

For $\mathrm{r}_{\mathrm{NW}} \sim 95 \mathrm{~nm}, \lambda \approx 35 \mathrm{~nm}$ was found considering a typical barrier of $\mathrm{V}_{\mathrm{b}} \sim 0.55 \mathrm{eV}$ [43], $\mathrm{n}_{0}=$ $4 \cdot 10^{17} \mathrm{~cm}^{-1}$ [10] (see supporting information), and the relative dielectric constant of $\mathrm{ZnO}\left(\varepsilon_{\mathrm{r}}=\right.$ 8.65 [44]). This $\lambda$ value is also in good correspondence with the literature [43].

In fact, this simple model explains the modulation of $\mathrm{G}$ in $\mathrm{N}_{2}$ and $\mathrm{SA}$ (up to a factor 2.5 [45]), but it fails with the huge change observed in PMMA-coated samples (factor 12). In this case, the contribution of the electrical mobility must be taken into account as well, since high mobility values have been reported in coated nanowires due to the reduction of electron scattering at their surfaces [19,38-40]. Following a methodology explained in detail elsewhere [10] (see supporting information), the electrical mobility of both coated and uncoated $\mathrm{ZnO}$ nanowires in SA was estimated $\left(\mu_{\text {(SA) }}=5 \pm 2 \mathrm{~cm}^{2} \mathrm{~V}^{-1} \mathrm{~s}^{-1}\right.$ and $\left.\mu_{\text {(PMMA) }}=53 \pm 8 \mathrm{~cm}^{2} \mathrm{~V}^{-1} \mathrm{~s}^{-1}\right)$. This difference (factor 10) explains the variation of $\mathrm{G}$ between the two experiments (see equations (2) and (3)).

Under illumination, UV photons generate electron-hole pairs in the bulk of the nanowires. After a few seconds, photoresponse $\left(\Delta \mathrm{G}_{\mathrm{ph}}\right)$ reaches a steady-state in which the recombination and the generation rates equal (figure 5.a and 5.b). Thus, the excess of $n$ and $p$ carriers $(\Delta n$ and $\Delta p)$ is given by [46],

$$
\Delta \mathrm{G}_{\mathrm{ph}} \propto \Delta \mathrm{n}=\Delta \mathrm{p}=\mathrm{g} \tau
$$

where $\mathrm{g}$ is the photogeneration rate of carriers per volume unit and $\tau$ their mean lifetime. If the number of recombination mechanisms is large, $\tau$ can be roughly estimated with the help of the Mathiessen's rule [46],

$$
\frac{1}{\tau}=\sum_{\mathrm{j}} \frac{1}{\tau_{\mathrm{j}}}
$$

Some authors claim the existence of two different mechanisms which steer the photoresponse in metal oxides $[28,29,47]$. The former one is a fast band-to-band recombination in their bulk with characteristic times in the nanosecond range (figure 5.a) [13]. The latter, which becomes dominant in nanosized materials [48], is highly dependent on the existence of chemisorbed oxygen molecules at their surfaces, since holes discharge oxygen species from the surface by indirect electron-hole recombination mechanisms (figure 5.a) [47]. Thus, equation (5) can be rewritten as,

$$
\Delta \mathrm{G}_{\mathrm{ph}} \propto \Delta \mathrm{n}=\Delta \mathrm{p}=\frac{\mathrm{g}}{1 / \tau_{\text {bulk }}+1 / \tau_{\text {surf }}}
$$

where $\tau_{\text {bulk }}$ and $\tau_{\text {surf }}$ are the lifetime of the photocarriers recombined in the bulk and at the surface. In oxygen-rich environments, surface recombination is favoured and lower $\Delta \mathrm{G}_{\mathrm{ph}}$ are produced. On the contrary, this mechanism is blocked in coated samples, explaining the experimental trend of measured photoresponses (figure 4),

$$
\Delta \mathrm{G}_{\mathrm{ph}(\text { PMMA) }}>\Delta \mathrm{G}_{\mathrm{ph}\left(\mathrm{N}_{2}\right)}>\Delta \mathrm{G}_{\mathrm{ph}(\mathrm{SA})} .
$$

When light is switched off, the two recombination mechanisms swiftly contribute to recover the initial carrier concentration in the nanowires. Nevertheless, the built-in potential near the surface caused by oxygen adsorption separates the photogenerated pairs: holes accumulate at the outer shell of the nanowire and electrons remain together in the inner part. This photogenerated effect prevents the recombination of a fraction of the pairs, whereby PPC is observed after the UV pulse (figure 5.c). Thereby $\Delta \mathrm{G} / \mathrm{G}_{0}$ will strongly depend on the oxygen content in air. That is to say, the higher this experimental parameter is the more efficient recombination through the surface and the less important the PPC becomes (figure 4.a). In the particular case of PMMAcoated samples, nanowires approaches to the so-called flat-band conditions [49] and thus, no charge separation takes place, favouring a complete recovery of the conductance baseline in dark (figure 4.b). 
If temperature increases, carriers gain thermal energy and they can easily overcome the built-in potential. In this case, PPC is not observed (figure 2).

According to first principles calculations, oxygen in air $\left(\mathrm{O}_{2}\right)$ undergoes a dissociative chemisorption at the surface oxygen vacant (VO) site in $\mathrm{ZnO}$ non-polarsurfaces [50,51], via filling the $\mathrm{VO}$ with one $\mathrm{O}$ atom originated from the adsorbate. This dissociation of $\mathrm{O}_{2}$ is exothermic and barrierless, and may even occur spontaneously at room temperature. This result suggests that $\mathrm{VO}$ are the surface sites at which oxygen assisted recombination of electrons and holes take place. From the point of view of the electron states, we demonstrated that VO sites are associated to surface states with energy $0.5 \mathrm{eV}$ above the valence band edge [52].

The here-proposed model only assumes the existence of a built-in potential to justify the charge pairs separation and hole accumulation near the surface of the material. Since this feature is common with other metal oxides, the model can be easily extended to other materials with similar PPC effects [53]. This model does not take into account the large number of bulk charge states located within the band gap of $\mathrm{ZnO}$ [35] with a strong influence in many applications, like gas sensing [35]. Other simplifications that may limit the model are those related to the flat band approximation in coated samples. These simplifications may prevent the explanation of the few-seconds kinetics to reach the steady state, which is experimentally observed even when the samples are coated with PMMA.
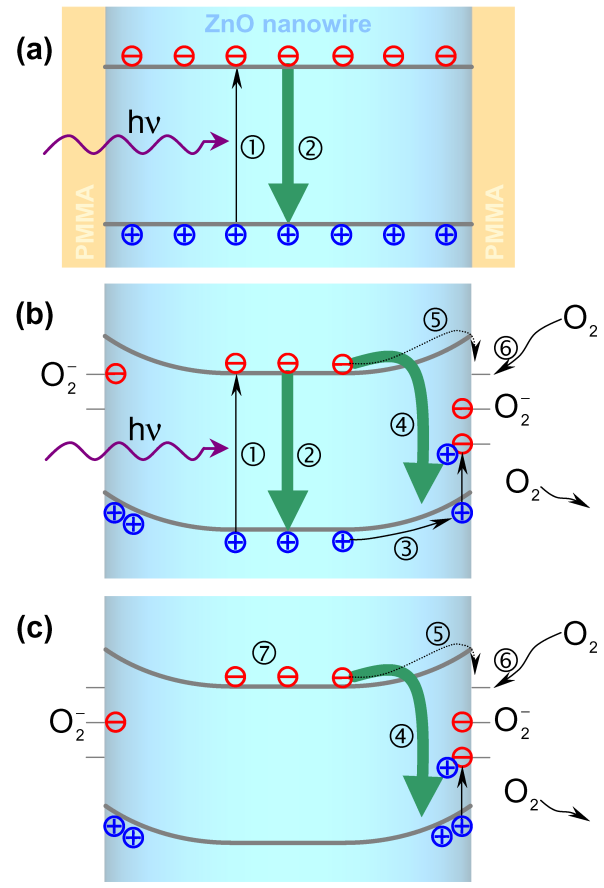

Figure 5. (a) Flat-band situation. When the material is illuminated with above-bandgap photons the (1) photogeneration of electron-hole pairs equals the (2) band-to-band recombination mechanism reaching steady conductance values. This case corresponds to the bulk material and also to the nanowires passivated with PMMA. (b) If band-bending near the surface is considered, an additional mechanism appears due to charge separation by this built-in potential: (3) holes tend to accumulate near the surface and recombine with electrons in an (4) oxygen-assisted surface recombination mechanism. (5) Temperature and (6) adsorption of oxygen by electron capture facilitates the access of the electrons to the surface. (c) When illumination is switched off, the bulk-like recombination mechanism rapidly extinguishes holes in the inner part of the nanowire. The remaining unpaired electrons are the responsible of the (7) persistent photoconductivity situation which can only be reverted by favouring the (4) oxygen-assisted surface mechanism. 


\section{Conclusions}

The study of Persistent PhotoConductivity (PPC) in individual $\mathrm{ZnO}$ nanowires demonstrated that the electrical transport properties in these nanomaterials are determined by the surface in two different ways. On the one hand, the effective mobility and the available free carriers $-\mu$ and $\mathrm{n}_{0^{-}}$are controlled by the surface recombination mechanisms assisted by the oxidizing molecules in air. On the other hand, the photoconductance $G$ is modified by the changes in the size of the depletion layer -?-.

In particular, PPC essentially depends on the built-in surface potential originated by the surface charges. Under UV illumination, this built-in potential induces electron-hole separation, and hole accumulation at the surface. Once light is switched off, accumulated charges need recombination paths to be drained and PPC appears if these paths are not available.

It is experimentally observed that PPC recovery times are linked with the availability of recombination paths. We observed that PPC was quickly reverted after increasing either the probing current -self-heating by Joule dissipation- or the oxygen content in air -favouring the surface recombination mechanisms. These experimental results and the proposed model helped us to elucidate the origin of the PPC in nanostructures. Thus, PPC can be completely blocked by heating the final devices or passivating their surfaces. It is noteworthy that PMMA coatings totally blocked PPC, overcoming one of the main limitations to integrate $\mathrm{ZnO}$ and other metal oxides in a new generation of optoelectronic nanodevices.

\section{Acknowledgments}

This work has been partially supported by the Spanish Ministry of Education and Science (MEC) through the projects n-MOSEN (MAT2007-66741-C02-01) and MAGASENS (NAN200409380-C04-01), and the UE and NanoSciEra Consortium through the project NAWACS (NAN2006-28568-E). JDP and RJD are indebted to the MEC for the FPU grant.

\section{References}

[1] Fan H J, Werner P and Zacharias M 2006 Small 2 700-17

[2] Law M, Goldberger J and Yang P 2004 Annu. Rev. Mater. Res. 34 83-122

[3] Wang Z L 2004 J. Phys.: Condens. Matter. 16 R829-58

[4] (a) Mathur S, Barth S, Shen H, Pyun J-C and Werner U 2005 Small 1 713-17 (b) Mathur S and Barth S 2007 Small 3 2070-5

[5] Chang P-C, Chien C-J, Stichtenoth D, Ronning C and Lu J G 2007 Appl. Phys. Lett. 90 113101

[6] Lieber C M and Wang Z L 2007 MRS Bull. 32 99-104

[7] Patolsky F, Timko B P, Zheng G and Lieber C M 2007 MRS Bull. 32 142-9

[8] Yang M-R, Chu S-Y and Chang R-C. 2007 Sens. Actuators B 122 269-73.

[9] Hernandez-Ramirez F, Tarancon A, Casals O, Rodriguez J, Romano-Rodriguez A, Morante J R, Barth S, Mathur S, Choi T Y, Poulikakos D, Callegari V and Nellen P M 2006 Nanotechnol. 17 5577-83

[10] Hernandez-Ramirez F, Tarancon A, Casals O, Pellicer E, Rodriguez J, RomanoRodriguez A, Morante J R, Barth S and Mathur S 2007 Phys. Rev. B 76085429

[11] Law M, Kind H, Messer B, Kim F and Yang P D 2002 Angew. Chem. Int. Ed. 41 2405-8

[12] Heo Y W, Norton D P, Tien L C, Kwon Y, Kang B S, Ren F, Pearton S J and LaRoche J R 2004 Mater. Sci. Eng. R 47 1-47.

[13] Wang J X, Sun X W, Wei A, Lei Y, Cai X P, Li C M, Dong Z L 2006 Appl. Phys. Lett. 88 233106

[14] Wang Z L and Song J H 2006 Science 312 242-6

[15] Kind H, Yan H, Messer B, Law M and Yang P 2002 Adv. Mater. 14 158-60

[16] Fan Z Y, Chang P C, Lu J G, Walter E C, Penner R M, Lin C H and Lee H P 2004 Appl. Phys. Lett. 85 6128-30

[17] Law J B K and Thong J T L 2006 Appl. Phys. Lett. 88133114 
[18] Soci C, Zhang A, Xiang B, Dayeh S A, Aplin D P R, Park J, Bao X Y, Lo Y H and Wang D 2007 Nano Lett. 7 1003-9

[19] Prades J D, Jimenez-Diaz R, Hernandez-Ramirez F, Fernandez-Romero L, Andreu T, Cirera A, Romano-Rodriguez A, Cornet A, Morante J R, Barth S and Mathur S $2008 \mathrm{~J}$. Phys. Chem. $C$ Article in press.

[20] Liu Y, Gorla C R, Liang S, Emanetoglu N, Lu Y, Shen H and Wraback M J $2000 \mathrm{~J}$. Electron. Mater. 29 69-74

[21] Lany S and Zunger A 2005 Phys. Rev. B 72035215

[22] Janotti A and Van de Walle C G 2005 Appl. Phys. Lett. 87122102

[23] Nayak J, Kasuya J, Watanabe A and Nozaki S 2008 J. Phys.: Condens. Matter 20195222

[24] Melnick D A 1957 J. Chem. Phys. 26 1136-46

[25] Sheinkman M K and Shik A Y 1976 Sov. Phys.-Semicond. 10 128-48

[26] Studenikin S A, Golego N and Cocivera M 2000 J. Appl. Phys. 87 2413-21

[27] Sharma P, Sreenivas K and Rao K V 2003 J. Appl. Phys. 93 3963-70

[28] Li Q H, Gao T, Wang Y G and Wang T H 2006 Appl. Phys. Lett. 86123117

[29] Reemts J and Kittel A 2007 J. Appl. Phys. 101013709

[30] Claflin B, Look D C and Norton D R 2007 J. Elect. Mater. 36 442-5

[31] ZnO nanowires were synthesized with a commercial Atomate's CVD system. The source material was 1:1 molar mixture of $\mathrm{ZnO}$ (metal basis $99.99 \%$ ) and graphite powder provided by Alfa Aesar. Crystalline nanowires with average radii $\left\langle\mathrm{r}_{\mathrm{NW}}\right\rangle=90+15 \mathrm{~nm}$ and lengths up to $\mathrm{L}=30$ were obtained [3]. High resolution TEM images showed wellfaceted single crystalline nanowires with dislocation free bodies grown along the [0001] direction (see supporting information).

[32] Hernandez-Ramirez F, Rodriguez J, Casals O, Russinyol E, Vila A, Romano-Rodriguez A, Morante J R and Abid M 2006 Sens. Actuators, B 118 198-203

[33] Seoul Optodevices model T9F34C

[34] Hernandez-Ramirez F, Prades J D, Tarancon A, Barth S, Casals O, Jiménez-Diaz R, Pellicer E, Rodriguez J, Juli M A, Romano-Rodriguez A, Morante J R, Mathur S, Helwig A, Spannhake J and Mueller G 2007 Nanotechnol. 18495501

[35] Özgür Ü, Alivov Ya I, Liu C, Teke A, Reshchikov M A, Dogan S, Avrutin V, Cho S-J and Morkoç H 2005 J. Appl. Phys. 98041301

[36] Look D C 1983 Semicond. Semimet. 19 75-170

[37] Prades J D, Jimenez-Diaz R, Hernandez-Ramirez F, Barth S, Cirera A, RomanoRodriguez A, Mathur S and Morante J R 2008 Appl. Phys. Lett. Submitted.

[38] Park W I, Kim J S, Yi G-C, Bae M H and Lee H J 2004 Appl. Phys. Lett. 85 5052-4

[39] He J H, Lin Y H, McConney M E, Tsukruk V V, Wang Z L and Bao G 2007 J. Appl. Phys. 102 084303-4

[40] (a) Hong W K, Sohn J I, Hwang, Kwon S-S, Jo G, Song S, Kim S-M, Ko H-J, Park S-J, Welland M E and Lee T 2008 Nano Lett. 8 950-6 (b) Hong W K, Kim B-J, Kim T-W, Jo G, Song S, Kwon S-S, Yoon A, Stach E A and Lee T, 2008 Colloids Surf. A: Physicochem. Eng. Aspects 313-314 378-82

[41] Arnold M S, Avouris P, Pan Z W and Wang Z L 2003 J. Phys. Chem. B 107 659-63

[42] Hernandez-Ramirez F, Prades J D, Tarancon A, Barth S, Casals O, Jimenez-Diaz R, Pellicer E, Rodriguez J, Morante J R, Juli M A, Mathur S and Romano-Rodriguez A 2008 Adv. Func. Mater. Article in press. DOI: 10.1002/adfm.200701191

[43] Comini E, Guidi V, Malagu C, Martinelli G, Pan Z, Sberveglieri G and Wang Z L 2004 J. Phys Chem 108 1882-87

[44] Bhargava B 1997 Properties of Wide Bandgap II-VI Semiconductors (London: Inspec)

[45] According to equation (2), the maximum conductance $\left(\mathrm{G}_{\mathrm{MAX}}\right)$ of the nanowire corresponds to the case of null depleted layer $(\lambda=0)$. The formation of a depleted layer of thichness $\lambda$ results in a decrease of the conductivity $\left(\mathrm{G}_{\mathrm{min}}\right)$. The change in the conductivity due to the formation of this depleted layer is thus $\mathrm{G}_{\mathrm{MAX}} / \mathrm{G}_{\min }=\left(\mathrm{r}_{\mathrm{NW}}\right)^{2} /\left(\mathrm{r}_{\mathrm{NW}}\right.$ $-\lambda)^{2}$. In our case, $\mathrm{r}_{\mathrm{NW}}=95 \mathrm{~nm}, \lambda=35 \mathrm{~nm}$ and $\mathrm{G}_{\mathrm{MAX}} / \mathrm{G}_{\min }=2.5$.

[46] Sze S M 1981 Physics of Semiconductor Devices (New York: John Wiley \& Sons, Inc) 
[47] Lin Y, Wang D, Zhao Q, Li Z, Ma Y and Yang M 2006 Nanotechnol. 17 2110-5

[48] The contribution of surface mechanisms is especially important in the case of nanowires because their diameters are significantly lower than the diffusion length of carriers in $\mathrm{ZnO}\left(\mathrm{L}_{\mathrm{ZnO}} \sim 1 \mu \mathrm{m}\right)[35]$.

[49] Surface passivation implies that there are not any gas molecules chemisorbed onto the $\mathrm{ZnO}$ surface [19,38-40]. In this situation, no charge is trapped at the nanowire' surface and no band-bending occurs. This obviously is a drastic approximation.

[50] An W, Wu X and Zeng X C 2008 J. Phys. Chem. C 112 5747-55

[51] Yan Y and Al-Jassim M M 2005 Phys. Rev. B 72235406

[52] Prades J D, Cirera A, Morante J R and Cornet A 2007 Thin Sol. Films 515 8670-3

[53] Geraldo V, Scalvi L V A, Morais E A, Santilli C V, Miranda P B and Pereira T J 2005 J. European Ceramic Soc. 25, 2825-28 


\section{A. Supporting information}

\section{A.1 Synthesis, morphology and structure of the $\mathrm{ZnO}$ nanowires}

$\mathrm{ZnO}$ nanowires were fabricated via a vapor-phase carbothermal transport process inside an Atomate's chemical vapour deposition (CVD) system. The source material was a 1:1 molar mixture of commercial $\mathrm{ZnO}$ (metal basis, 99.99\%) and graphite powder (crystalline, 300 mesh, 99\%) from Alfa Aesar. Gold nanoparticles were used as catalytic islands on amorphous alumina substrates. Uniform and crystalline nanowires were obtained with mean radius $\left\langle\mathrm{r}_{\mathrm{NW}}\right\rangle=90 \pm 15$ $\mathrm{nm}$ and lengths up to $\mathrm{L}=30 \mu \mathrm{m}$ (Figure I). High resolution TEM images showed well-faceted single crystalline nanowires with dislocation free bodies grown along the [0001] direction (Figure II).

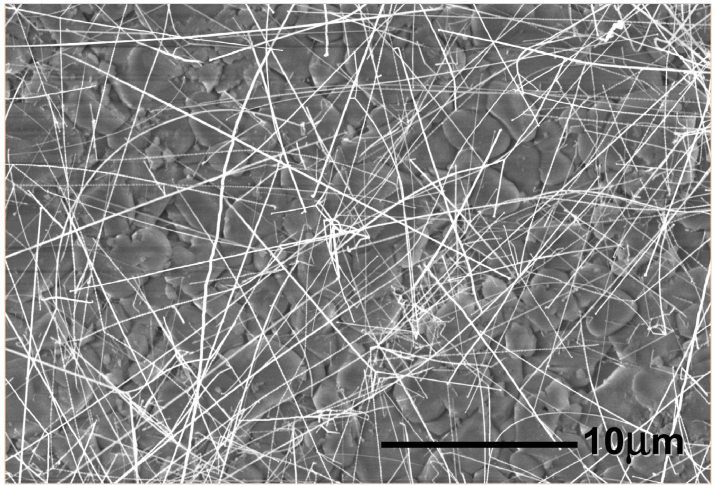

Figure I. SEM micrograph of the as grown $\mathrm{ZnO}$ nanowires. Nanostructures with mean radius $r_{N W}=90 \pm 15 \mathrm{~nm}$ and lengths up to $30 \mu \mathrm{m}$ were obtained.

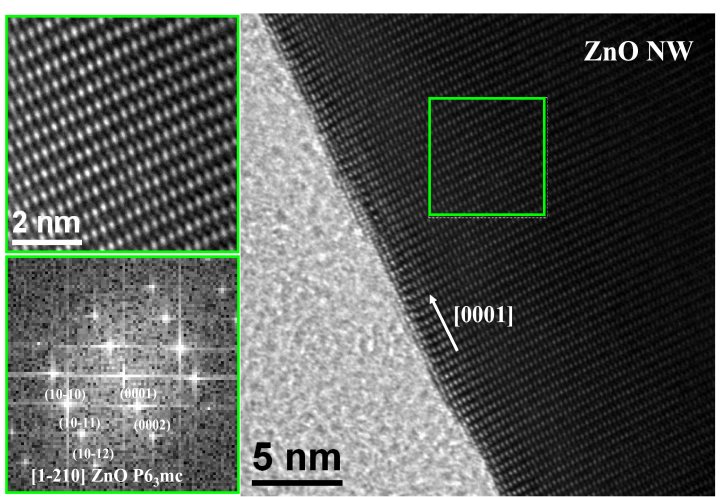

Figure II. HRTEM analysis of a $\mathrm{ZnO}$ nanowire. Crystalline and dislocation-free $\mathrm{ZnO}$ grow along the [0001] direction. Surface roughness is less than 3 monolayers. Thanks are due to Dr. J. Arbiol.

\section{A.2 Electrical $I(V)$ characteristics}

The nanowires' $I(V)$ characteristics measured in 2 and 4-probe configurations are shown in figure III. In the 2-probe case, we observed a symmetric non-linear characteristic corresponding to two back-to-back Schottky diodes in series with the resistance of the nanowire $(1 / \mathrm{G})$. The conduction through the metal-semiconductor $(\mathrm{Pt} / \mathrm{ZnO})$ junction is properly described by thermoionic emission and interface states assisted tunnelling (TE+TuSA) [a,b]. According to this model, the voltage drop across the hole structure in 2-probe configuration $\left(\mathrm{V}_{(2-\mathrm{p})}\right)$ is $[\mathrm{a}, \mathrm{b}]$

$$
V_{(2-p)}=V_{\text {diode }}+V_{N W}=\left(\frac{\ln I-\beta}{\alpha(n)}\right)^{4}+\frac{I}{G(n, \mu)}
$$

where $I$ is the current, $G$ is the conductance of the nanowire and $\alpha$ and $\beta$ are parameters of the TE+TuSA model for the voltage drop at the reverse biased diode $\left(\mathrm{V}_{\text {diode }}\right)$. It is noteworthy that $\alpha$ and $\mathrm{G}$ are analytic functions dependent on the free carriers concentration $\mathrm{n}$ and the mobility $\mu$. Thus, fitting the experimental curve to equation (i) it is possible to estimate $\mathrm{G}$ and also decouple $\mathrm{n}$ and $\mu$. A detailed explanation of this procedure can be found elsewhere [a]. In dark $\left.{ }_{0}\right)$ and synthetic air (SA), the conductance of our nanowires was $\mathrm{G}_{0(\mathrm{SA})}=60 \pm 8 \mathrm{nS}$ and the correspondent carrier concentration and mobility were $\mu_{(\mathrm{SA})}=5 \pm 2 \mathrm{~cm}^{2} \mathrm{~V}^{-1} \mathrm{~s}^{-1}$ and $\mathrm{n}_{0}=4 \cdot 10^{17} \mathrm{~cm}^{-1}$.

In the case of 4-probe measurements, we observed a linear characteristic only due to the resistance of the nanowire. From the slope of this plot we estimated the conductance in dark of the nanowire in $\mathrm{G}_{0(\mathrm{SA})}=59 \pm 6 \mathrm{nS}$ which was in accordance with the previous estimation. The measurements reported in the main text were performed in 4-probe configuration to avoid the interfering effect form the contacts. 


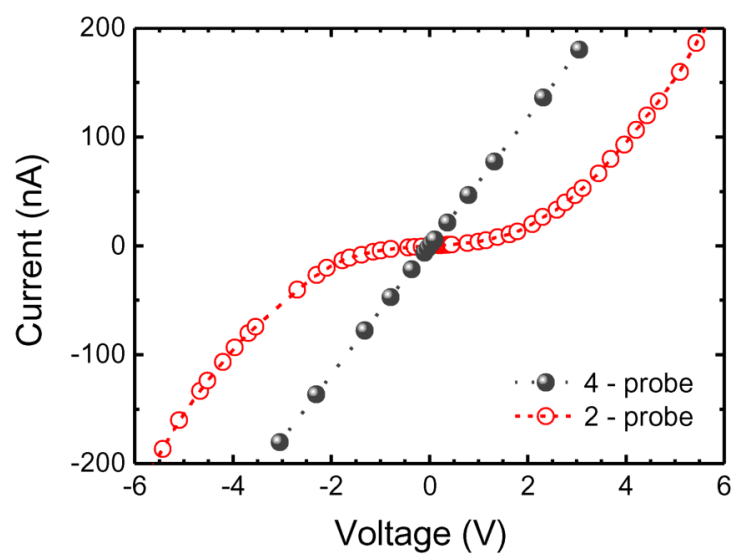

Figure III. I(V) characteristics of a single $\mathrm{ZnO}$ nanowire acquired in 2 and 4-probe configurations in dark conditions. Lines represent the mathematical fitting to estimate the values described in the text.

\section{A.3 Wavelength photoresponse threshold in $\mathrm{ZnO}$ nanowires}

To stimulate the photoresponse in $\mathrm{ZnO}$ nanowires, we used a set of LEDs [c] of energies hv above and below the bandgap of this semiconductor $\left(\mathrm{E}_{\text {gap }}(\mathrm{ZnO})=3.37 \mathrm{eV}\right.$ [d]). In figure IV we show the fully-ohmic $\mathrm{I}(\mathrm{V})$ characteristics under illumination at different energies. For hv below the $\mathrm{E}_{\text {gap }}(\mathrm{ZnO})$, photocurrent increase in less than $5 \%$, while for $\mathrm{hv}$ above $\mathrm{E}_{\text {gap }}(\mathrm{ZnO})$, the photoresponse is about $160 \%$. This indicates that photoconduction is essentially due to band-toband electron-hole pairs generations. From the technological point of view, this results also suggest that $\mathrm{ZnO}$ nanowires are almost visible-blind $\mathrm{UV}$ photodetectors with flat response in the near-UV region. For this reason, we centre our study in the source at hv $=3.64 \mathrm{eV}(340 \mathrm{~nm})$.

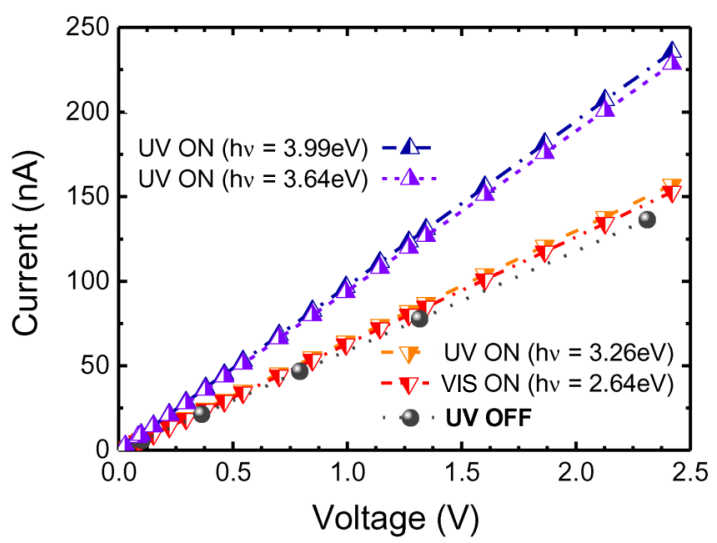

Figure IV. 4-probe $\mathrm{I}(\mathrm{V})$ characteristics of a single $\mathrm{ZnO}$ nanowire at different illumination conditions. Significant photoresponse is only obtained with photon energies above $\mathrm{E}_{\mathrm{gap}}(\mathrm{ZnO})=3.36 \mathrm{eV}$. All measurements were acquired under photon flux $\Phi_{\mathrm{ph}}=3.3 \cdot 10^{18} \mathrm{~m}^{-2} \mathrm{~s}^{-1}$.

\section{A.4 UV transmittance and conductance of PMMA layers}

To evaluate the transparency of PMMA to UV light, transmittance spectroscopy experiments were performed on PMMA layers of thickness $(475 \pm 50) \mathrm{nm}$ deposited over fused silica substrates (figure V). In figure VI, the transmittance spectrum of one of these layers is shown, after subtracting the silica contribution. Transmittances values above $90 \%$ were obtained for wavelengths from $250 \mathrm{~nm}$ to $800 \mathrm{~nm}$. 
Chips with pre-patterned microelectrodes were also coated in order to estimate the conductance and photoresponse of these PMMA layers. Typical conductance values were always below $0.1 \mathrm{nS}$, and any significant change was observed after UV illumination (figure VII).
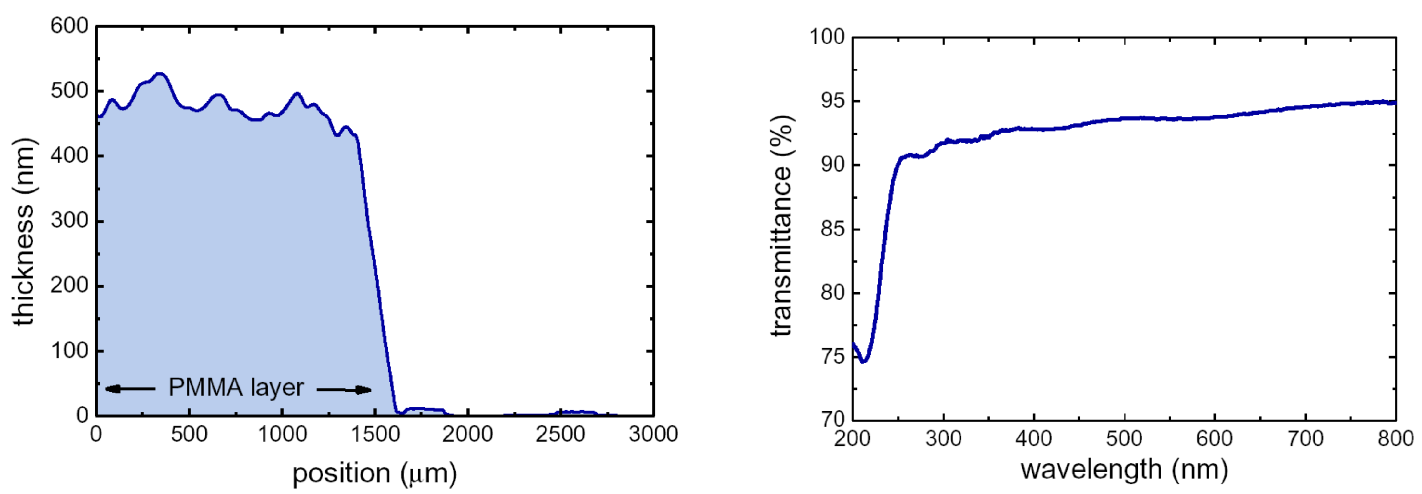

Figure V. Perfilometry profile of a cracked layer of Figure VI. Transmittance spectrum of a PMMA layer. PMMA deposited onto a silica substrate. Contribution from the silica substrate was corrected.

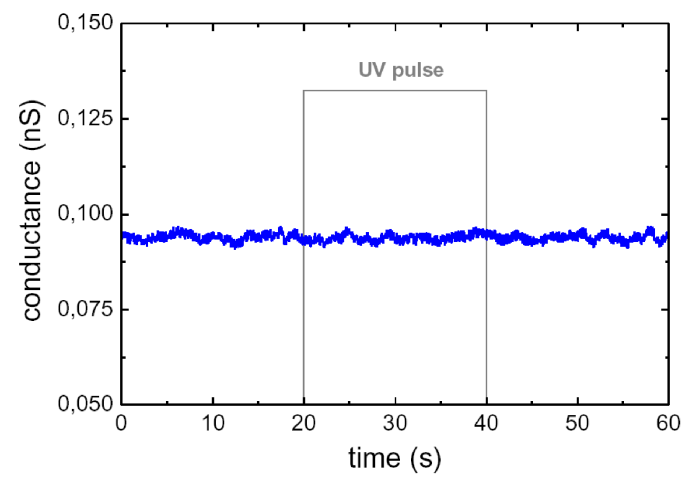

Figure VII. 4-probe measurement of the conductance of a PMMA layer. No response to UV light was observed. 


\section{A.4 References:}

[a] Hernandez-Ramirez F, Tarancon A, Casals O, Pellicer E, Rodriguez J, RomanoRodriguez A, Morante J R, Barth S and Mathur S 2007 Phys. Rev. B 76085429

[b] Zhang Z, Yao K, Liu Y, Jin C, Liang X, Chen Q, Peng L-M 2007 Adv. Func. Mater. 17 2478-89

[c] We used the following light emitting diodes from Seoul Optodevices: T9F31C $(310 \pm 10 \mathrm{~nm}), \quad$ T9F34C $(310 \pm 10 \mathrm{~nm}), \quad$ UVLED380-10 $(380 \pm 15 \mathrm{~nm})$ and L5T15B $(470 \pm 5 \mathrm{~nm})$.

[d] Özgür Ü, Alivov Ya I, Liu C, Teke A, Reshchikov M A, Dogan S, Avrutin V, Cho S-J and Morkoç H 2005 J. Appl. Phys. 98041301 